\title{
COMUNICACIÓN
}

\section{Presencia de ooquistes de Cryptosporidium spp en aguas superficiales en Costa Rica}

\author{
SILENIA LUNA, LILIANA REYES L., MISAEL CHINCHILLA y GABRIELA CATARINELLA
}

\section{PRESENCE OF Cryptosporidium SPP OOCYSTS IN SURFACE WATER IN COSTA RICA}

The presence of Cryptosporidium spp oocysts was determined in seven samples of crude surface water and seven of treated non-chlorinated water from a water plant in San José,Costa Rica. Concentration and staining techniques were used to demonstrate the presence of parasites. Cryptosporidium spp oocysts were found in six of seven untreated water samples (85.7\%) and in four of the seven treated non-chlorinated samples (57\%). The role of water as a transmission vehicle for this parasite in Costa Rica is discussed.

Key words: Protopzoa, Epidemiology, Cryptosporidium, Costa Rica.

\section{INTRODUCCIÓN}

Cryptosporidium spp son coccidios parásitos que infectan tanto seres humanos como animales y son trasmitidos por ooquistes resistentes a las condiciones ambientales ${ }^{1}$. De las especies conocidas solamente $C$. parvum parece estar asociada con infección humana ${ }^{1}$. Este organismo presenta distribución mundial ${ }^{2}$ y su transmisión ha sido relacionada a la ingestión de aguas contaminadas con materia fecal $^{3,4}$, a la ingestión de alimentos contaminados ${ }^{5} \mathrm{y}$ a la transmisión persona a persona ${ }^{6}$. Por métodos fenotípicos y genotípicos se ha demostrado la presencia de dos tipos de $C$. parvum: el genotipo 1 ocurre exclusivamente en personas natural- mente infectadas y el genotipo 2 presente en ganado vacuno y también en personas y por lo tanto responsable de la transmisión zoonótica de la enfermedad ${ }^{7,8}$. La contaminación de aguas de consumo con material fecal de ganado vacuno así como con aguas negras o material fecal humana se han asociado con brotes de diarrea causada por este protozoario $^{9,10}$. Es importante además el considerar que los métodos usuales de tratamiento de aguas de consumo, incluida la desinfección química o los métodos de filtración, no son eficientes en la remoción de los ooquistes de este protozoario ${ }^{11,12}$. Por ser Cryptosporidium una causa de diarrea infantil en Costa Rica $^{13}$ se consideró importante estudiar la presencia de este parásito en aguas

\footnotetext{
* Departamento de Parasitología, Facultad de Microbiología, Universidad de Costa Rica Instituto Costarricense de Acueductos y Alcantarillados, Costa Rica.

Correspondencia: Dr. Misael Chinchilla, Facultad de Microbiología, Universidad de Costa Rica
} 
superficiales en nuestro país.

\section{MATERIAL Y MÉTODOS}

Se colectaron un total de 14 muestras de agua; 7 de agua cruda a su momento de entrada a la planta de tratamiento y 7 de agua tratada por filtración floculación y sedimentación pero antes de su cloración. La planta de tratamiento estudiada pertenece al Instituto Nacional de Acueductos y Alcantarillados de Costa Rica, recibe agua de los ríos Virilla y Coronado los cuales fluyen por fincas ganaderas y lecheras y también por zonas altamente pobladas antes de ingresar al tratamiento. Se filtraron muestras de agua de aproximadamente 400 litros cada una utilizando filtros de polipropileno, con un poro de $1 \mu \mathrm{m}$ (Balstron Company, LTD Maindstone, Inglaterra Code 100-12-C) $)^{14}$, colocados en un soporte para filtros con una llave de entrada y una de salida para la toma de la muestra. Se colocó además un medidor de agua para estimar el volumen filtrado. El muestreo se realizó entre julio de 1997 y Febrero 1998, incluyendo muestras en la estación seca y la lluviosa.

Aislamiento e identificación de los ooquistes: la matriz del filtro se cortó con una tijera, se separó en tiras de aproximadamente 2-5 x $2 \mathrm{~cm}$ y posteriormente se eluyó con 4 litros de agua destilada al $0,1 \%$ de Tween 80 . La suspensión resultante se centrífugo y el sedimento se resuspendió en una gradiente de sucrosa $(1,18 \mathrm{sp} \text {. gr. })^{14}$ en la que los ooquistes flotan. La capa superficial fue colectada y lavada va- rias veces con agua destilada, el último sedimento fue utilizado para realizar extensiones y tinción de Koster modificada ${ }^{15}$.

Para la identificación de los ooquistes se utilizaron los siguientes criterios: tamaño (4-6 $\mu \mathrm{m})$, morfología, dobleces en la pared del ooquiste y presencia de cuatro esporozoitos ${ }^{9}$.

Además, para determinar la calidad del agua, una porción de ella fue utilizada para la determinación de coliformes fecales y totales expresados como número más probable $(\mathrm{NMP})^{16}$.

\section{RESULTADOS Y DISCUSIÓN}

Seis de las siete muestras de agua cruda $(85,7 \%)$ y cuatro de las siete de agua tratada $(57 \%)$ presentaron ooquistes. Cinco muestras fueron positivas durante la estación lluviosa y cinco durante la estación seca. Los números de coliformes totales fueron elevados en las aguas crudas, de 15.000-110.000 NMP/100 ml (promedio 83.000 NMP/ $100 \mathrm{ml}$ ). Los coliformes fecales fueron también elevados, 2.400 a $110.000 \mathrm{NMP} / 100 \mathrm{ml}$ (promedio 31.776 NMP/ $100 \mathrm{ml}$ ). Las aguas post- tratamiento presentaron niveles bajos de coliformes totales (negativas o 9,1-210 NMP/100 ml) y también presentaron niveles bajos de coliformes fecales (negativas o 7,3-9,1 NMP/100 ml) (Tabla 1). Como se mencionó con anterioridad las muestras de agua tratada fueron tomadas antes del proceso de cloración lo que explica las bajas concentraciones de coliformes tanto totales como fecales. En Costa Rica la criptosporidiosis ha sido informada tanto en la estación lluviosa como en

Tabla 1. Control microbiológico de planta de tratamiento durante el periodo de estudio.

Mes

Agua cruda

Agua tratada**

Coliformes totales* Coliformes fecales* Coliformes totales* Coliformes fecales*

\begin{tabular}{|c|c|c|c|c|}
\hline Julio & 110.000 & 4.300 & 43 & 7,3 \\
\hline Agosto & 15.000 & 2.400 & 210 & 9,1 \\
\hline Setiembre & 110.000 & 110.000 & 9,1 & Negativa \\
\hline Octubre & 46.000 & 3.900 & Negativa & Negativa \\
\hline Noviembre & 110.000 & 24.000 & Negativa & Negativa \\
\hline Diciembre & 110.000 & 46.000 & 23 & Negativa \\
\hline
\end{tabular}

* NMP/100 ml

** Filtrado previa cloración 
la seca con un máximo durante la estación lluviosa (mayo a noviembre) ${ }^{13}$. Igualmente ooquistes de Cryptosporidium spp han sido aislados de muestras de vegetales frescos en nuestro país en la estación seca y en la lluviosa ${ }^{17}$.

En este estudio se demuestra la presencia de ooquistes independientemente de las limitaciones propias de los procedimientos utilizados; al respecto, se indica que los métodos de aislamiento presentan una baja tasa de recuperación y detección, ya que hasta un $85 \%$ de los ooquistes son retenidos dentro del filtro 9 . Sin embargo, el procesamiento y la clarificación resultan en una pérdida del $90 \%$ de los mismos.

En conclusión, se informa que la presencia de ooquistes de Cryptosporidium en aguas superficiales es independiente de la estación del año. Además, como se ha demostrado en otros países $^{9,18}$, los métodos usuales de tratamiento de agua no son completamente eficientes en la remoción de los ooquistes. Se establece la posibilidad de transmisión de esta parasitosis por la ingestión de agua en nuestro país.

\section{RESUMEN}

Se determinó la presencia de ooquistes de Cryptosporidium spp en aguas superficiales en una planta de tratamiento en San José Costa Rica. Se analizaron siete muestras de aguas pre-tratadas y siete de aguas tratadas no cloradas. Se utilizaron técnicas de concentración y tinción para demostrar la presencia de los ooquistes. Seis de las siete muestras de agua pre- tratadas y cuatro de las siete de agua tratadas fueron positivas por ooquistes de Cryptosporidium. Se discute el papel del agua como fuente de infección en Costa Rica.

\section{REFERENCIAS}

1.- TZIPORI S, GRIFFITHS J. Natural history and biology of Cryptosporidium parvum. Adv Parasitol 1998; 40: 5-35.

2.- CURRENT W L, GARCIA L S. Cryptosporidiosis. Clin Microbiol Rev 1991; 4: 325-58.
3.- ONGERTH J E, STIBBS H H. Identification of Cryptosporidium oocysts in river water. Appl Environ Microbiol 1987; 53: 672-6.

4.- MAC KENZIE W $\mathrm{R}$ et a1. A massive outbreak in Mi1waukee of Cryptosporidium infection transmitted through the public water supply. $\mathrm{N}$ Engl J Med 1994; 331: 161-7.

5.- ROSE J B, SLIFKO T R. Giardia, Crytosporidium, and Cyclospora and their impact on foods: a review. J Food Prot 62: 1059-070.

6.- TANGERMANN R H, GORDON S, WISNER P, KRECKMAN L. An outbreak of cryptosporidiosis in a day-care center in Georgia. Am J Epidemiol 1991; 133: 471-6.

7.- MCLAUGHLIN J, AMAR C, PEDRAZA-DIAZ S, NICHOLS G L. Molecular epidemiological analysis of Cryptosporidium spp. In the United Kingdom: results ofgenotyping Cryptosporidum spp. in 1.705 feca1 samples from humans and 105 fecal samples from livestock animals. J Clin Microbiol 2000; 38: 3984-90.

8.- CORINNE S L et al. Molecular epidemiology of cryptosporidiosis outbreaks and transmission in British Columbia, Canada. Am J Trop Med Hyg 1999; 61: 63-9.

9.- SMITH H V, ROSE B. Waterborne cryptosporidiosis. Parasitol Today 1999; 6: 8-12.

10.- LEVY D A et al. Surveillance for waterborne diseases outbreaks United States, 1995-1996. Mor. Mortal. Wk1y. Rep 1998; 47: 1-34.

11.- WIDMER G, CARRAWAY M, TZIPORI S. Waterborne Cryptosporidium: a perspective from the USA. Parasitol Today 1996; 12: 286-90.

12.- GUERRANT R L. Cryptosporidiosis: an emerging, highly infectious threat. Emerg Infect Dis 1997; 3: 130.

13.- MATA L. Cryptosporidium and other protozoa in diarrhea1 disease in less developed countries. Ped Infect Dis 1986; 5: s117-s30.

14.- SMITH H V. Intestinal protozoa. En. Medica1 Parasitology a practical approach. In: S H Gi11espie y P M Hawkey (eds). Oxford University Press, London, U.K., 1995. p 102-10.

15.- KAGEMURA P, BRANDT J, TAELMAN $\mathrm{H}$, JONAS C. Modified staining method for the diagnosis of cryptosporidiosis. Ann Soc Belge Med Trop 1984; 64: 171-5.

16.- ANONYMOUS. Standard Methods for the examination of water and wastewater. 18th Edition. APHA Washington D.C. 1992.

17.- MONGE R, CHINCHILLA M. Presence of Cryptosporidum oocysts in fresh vegetables. J Food Prot 1996; 59: 202-3.

18.- PERZ J F, ENNEVER F K, LE BLANCQ S M. Crytosporidium in tap water. Comparison of predicted risks with observed levels of disease. Am J Epidemiol 1998; 147: 289- 301. 